\title{
Disfunções do assoalho pélvico pós radioterapia para tratamento do carcinoma de colo uterino: uma revisão integrativa
}

Post-radiation pelvic floor dysfunctions for the treatment of cervical cancer: an integrative review

Disfunciones del suelo pélvico posteriores a la radiación para el tratamiento del cáncer de cuello uterino: una revisión integradora

Recebido: 19/10/2021 | Revisado: 27/10/2021 | Aceito: 02/11/2021 | Publicado: 04/11/2021

Laís Cabral de Lima

ORCID: https://orcid.org/0000-0002-6866-5071

Universidade Católica de Pernambuco, Brasil

E-mail: laiscabra167@gmail.com

Thaynan Santos da Silva

ORCID: https://orcid.org/0000-0001-6879-2591

Universidade Católica de Pernambuco, Brasil

E-mail: thaynansantos10@hotmail.com

Amanda Santos Vidal de Negreiros

ORCID: https://orcid.org/0000-0002-2024-0277

Universidade Católica de Pernambuco, Brasi

E-mail: amandavidaldengreiros@gmail.com

Ana Carolina Queiroz Vieira

ORCID: https://orcid.org/0000-0002-9477-6138

Universidade Católica de Pernambuco, Brasil E-mail: ana.2017205774@unicap.br

Samuel Cabral de Lima

ORCID: https://orcid.org/0000-0001-8173-5620

Faculdade Pernambucana de Saúde, Brasil

E-mail: scabraldelimas@gmail.com

Silvana Maria de Macêdo Uchôa

ORCID: https://orcid.org/0000-0002-4635-9428 Universidade Católica de Pernambuco, Brasil E-mail: silvanammu@gmail.com

Érica Patrícia Borba Lira Uchôa

ORCID: https://orcid.org/0000-0003-4099-1876 Universidade Católica de Pernambuco, Brasil E-mail: ericaluchoa@gmail.com

Valéria Conceição Passos de Carvalho

ORCID: https://orcid.org/0000-0001-8314-9000 Universidade Católica de Pernambuco, Brasil E-mail: valeriapassos@gmail.com

\begin{abstract}
Resumo
Objetivo: Verificar a literatura acerca das disfunções do assoalho pélvico promovidas pela radioterapia após o tratamento de carcinoma de colo uterino. Métodos: Revisão integrativa da literatura, na qual foi feita uma verificação de bibliografias publicadas nas bases de dados Pubmed, Scielo, Portal Capes, Lilacs, Cochrane, PEDro e Medline, no período de 2010 a 2021. As palavras-chave utilizadas foram "disfunções", "assoalho pélvico", "radioterapia" e "neoplasias do colo do útero" e suas correlatas na língua inglesa e espanhola. Resultados: No final da análise, sete estudos foram incluídos por se adequarem aos critérios da pesquisa, em que foi verificado que as disfunções pélvicas mais relatadas na literatura após a radioterapia para tratamento de câncer de colo de útero são a dispareunia e a incontinência urinária. Considerações finais: Foi possível inferir que esse tipo de intervenção traz inúmeros impactos para o assoalho pélvico, com destaque para a incontinência urinária e a dispareunia. Sugere- a inserção de forma mais ampla de atividades de recuperação através da fisioterapia para as alterações decorrentes desse tipo de tratamento, visando a melhora da funcionalidade dos músculos do assoalho pélvico e redução das alterações pélvicas, que trazem complicações no bem-estar físico e psicológico dessa população.
\end{abstract}

Palavras-chave: Distúrbios; Diafragma pélvico; Braquiterapia; Teleterapia; Colo do útero.

\section{Abstract}

Objective: To verify the literature on pelvic floor disorders caused by radiotherapy after the treatment of cervical cancer. Methods: An integrative literature review, in which bibliographies published in Pubmed, Scielo, Portal Capes, 
Lilacs, Cochrane, PEDro and Medline databases were checked, from 2010 to 2021. The keywords used were "dysfunctions", "pelvic floor", "radiotherapy" and "neoplasms of the cervix" and their correlates in English and Spanish. Results: At the end of the analysis, seven studies were included for meeting the research criteria, in which it was found that the most reported pelvic dysfunctions in the literature after radiotherapy for the treatment of cervical cancer are dyspareunia and urinary incontinence. Final considerations: It was possible to infer that this type of intervention has numerous impacts on the pelvic floor, especially urinary incontinence and dyspareunia. It suggests the broader insertion of recovery activities through physiotherapy for changes resulting from this type of treatment, aiming at improving the functionality of the pelvic floor muscles and reducing pelvic changes, which bring complications to physical and psychological well-being of this population.

Keywords: Disorders; Pelvic diaphragm; Brachytherapy; Teletherapy; Cervix.

\section{Resumen}

Objetivo: Verificar la literatura sobre las alteraciones del suelo pélvico provocadas por la radioterapia tras el tratamiento del cáncer de cuello uterino. Métodos: Revisión integrativa de la literatura, en la que se revisaron las bibliografías publicadas en las bases de datos Pubmed, Scielo, Portal Capes, Lilacs, Cochrane, PEDro y Medline, de 2010 a 2021. Las palabras clave utilizadas fueron "disfunciones", "suelo pélvico", "radioterapia", "Y" neoplasias del cuello uterino" y sus correlatos en inglés y español. Resultados: Al final del análisis, se incluyeron siete estudios por cumplir con los criterios de investigación, en los cuales se encontró que las disfunciones pélvicas más reportadas en la literatura después de la radioterapia para el tratamiento del cáncer de cuello uterino son la dispareunia y la incontinencia urinaria. Consideraciones finales: Se pudo inferir que este tipo de intervención tiene numerosos impactos en el suelo pélvico, especialmente la incontinencia urinaria y la dispareunia. Sugiere la inserción más amplia de actividades de recuperación a través de la fisioterapia para los cambios resultantes de este tipo de tratamiento, con el objetivo de mejorar la funcionalidad de la musculatura del suelo pélvico y reducir los cambios pélvicos, que traen complicaciones al bienestar físico y psicológico de esta población.

Palabras clave: Transtornos; Diafragma pélvico; Braquiterapia; Teleterapia; Cérvix.

\section{Introdução}

O câncer é um dos principais problemas de saúde pública do mundo, estando entre as quatro principais causas de morte precoce antes dos 70 anos. Estima-se que no triênio de 2020-2022, o Brasil tenha cerca de 16.590 novos casos de câncer de colo de útero a cada 100 mil habitantes, ficando atrás estatisticamente apenas do câncer de mama (66.280) e do câncer de colorretal (20.470) na população feminina, excluindo o câncer de pele não melanoma (Inca, 2020). A infecção pelos tipos oncogênicos do papilomavírus humano (HPV) pode ser apontada como um dos principais fatores causais dessa neoplasia. Existem também distintas condições que favorecem o seu desenvolvimento, como outras doenças sexualmente transmissíveis, idade, multiparidade, tabagismo e uso prolongado de contraceptivos (Castaneda \&, 2018; Fonseca \&, 2010).

Os procedimentos terapêuticos básicos utilizados para esse tipo de câncer são a cirurgia e a radioterapia, nos casos de diagnóstico precoce, e a quimioterapia, naqueles casos de diagnóstico mais tardio. Há um destaque na utilização da radioterapia, sendo possível distinguir duas facetas muito utilizadas: a braquiterapia e a teleterapia. Apesar disso, as duas modalidades podem trazer sequelas para as pessoas submetidas a essa terapêutica, com destaque principalmente para as disfunções do assoalho pélvico e suas consequentes implicações na qualidade de vida. (Bernardo \&, 2007; Einstein \&, 2012; Freire \&, 2010). O assoalho pélvico é constituído por diversas estruturas, dentre as quais se sobressaem a musculatura, fáscias e ligamentos, localizados logo abaixo da pelve e dentre as funções dessas estruturas, destacamos o seu papel na continência urinária e fecal, no auxílio na passagem do feto durante o trabalho de parto e na função sexual. (Ferla \&, 2016; Souza \&, 2017).

Após o tratamento os principais sistemas afetados nas pacientes oncológicas são o endócrino, urinário e reprodutivo. Considerando que essa neoplasia não é uma doença de curta duração, são necessários maiores cuidados com a funcionalidade destas mulheres, para prevenir futuras incapacidades, sendo importante destacar como repercussões a gama de alterações vesicais promovidas pela radioterapia, destacando-se a incontinência urinária, incontinência fecal e a bexiga hiperativa. Além desses transtornos, essas pacientes também apresentam outras disfunções como a dispareunia, problemas sensoriais, falta de lubrificação vaginal, linfedema de membro inferiores, estenose vaginal, micção obstrutiva e alterações no padrão evacuatório (Osann \&, 
É fundamental que essas pacientes sejam vistas de forma inclusiva, devendo ser efetuada a avaliação das prováveis disfunções pélvicas que irão surgir, e para tal faz-se necessário um exame físico funcional mais detalhado pelo fisioterapeuta, a fim de tentar minimizar presumíveis efeitos negativos na qualidade de vida dessas pacientes (Bernardo \&, 2007; Oliveira \&, 2005). Alguns estudos apontam que cerca de $80 \%$ das pacientes submetidas a radioterapia apresentam algum tipo de disfunção sexual, com destaque para estenose vaginal ou encurtamento em cerca de $64,9 \%$ das mulheres, além de relatos sobre a falta de lubrificação e dor na relação sexual (Corrêa \&, 2016; Ros \&, 2016).

O presente estudo tem como objetivo verificar a literatura acerca das disfunções do assoalho pélvico promovidas pela radioterapia após o tratamento de carcinoma de colo uterino, bem como identificar qual a disfunção mais prevalente após a radioterapia para o carcinoma, a faixa etária mais acometida e identificar nos questionários de qualidade de vida em qual domínio ocorre mais alterações.

\section{Metodologia}

Este projeto está vinculado a Universidade Católica de Pernambuco ao Centro de Ciências Biológicas e Saúde e ao curso de Fisioterapia. Está vinculado projeto de pesquisa intitulado "Abordagem fisioterapêuticas nas disfunções sexuais femininas e masculinas", sob coordenação de Silvana Maria Macedo Uchôa, com número de CAAE "03239318.1.0000.5206”, já aprovado pelo comitê de ética e pesquisa em seres humanos, com número de parecer: 3.049 .749 e pertencente ao grupo de Fisioterapia baseada em Evidências.

O tipo de estudo é uma revisão integrativa da literatura na qual está sendo feita uma análise de artigos publicados nas bases eletrônicas de dados Pubmed, Scielo, Portal Capes, Lilacs, Cochrane, PEDro e Medline, no período de 2010 a 2020. Utilizando-se as seguintes palavras-chave: "Disfunções", "Assoalho pélvico", "Radioterapia" e "Neoplasias do colo do útero" e suas correspondentes na língua inglesa e espanhola. Para o critério de inclusão foram utilizados Artigos que abordem na sua temática as consequências da radioterapia pós carcinoma de colo de útero no assoalho pélvico, artigos publicados no período de 2010 a 2021, artigos que se encaixem até o nível 4 da revisão integrativa, artigos nas línguas português, inglês e espanhol. Foram excluídos artigos que não estejam indexados em bases de dados científicas, teses e dissertações de mestrado, relatos de caso e revisões de literatura sem meta análise, sites ou páginas eletrônicas sem embasamento científico e artigos que se encaixam nos níveis 5 e 6 da análise crítica da revisão integrativa.

Após a busca nas bases eletrônicas de dados citadas foi feita uma leitura do título e do resumo, em seguida os estudos passaram por uma análise crítica daqueles que foram incluídos. Esses estudos foram divididos em seis níveis, o que tornou possível a validação da metodologia e dos resultados obtidos em cada pesquisa. Tal fato, corrobora com a prática baseada em evidências que cada vez mais se faz necessária na área da fisioterapia. Esses níveis determinam uma hierarquia das evidências encontradas segundo as características da pesquisa. A classificação é elaborada da seguinte forma: nível 1: evidências resultantes da meta-análise de múltiplos estudos clínicos controlados e randomizados; nível 2: evidências obtidas em estudos individuais com delineamento experimental; nível 3: evidências de estudos quase-experimentais; nível 4: evidências de estudos descritivos (não-experimentais) ou com abordagem qualitativa; nível 5: evidências provenientes de relatos de caso ou de experiência; nível 6: evidências baseadas em opiniões de especialistas (Souza \&, 2010).

Para a obtenção dos resultados os dados encontrados foram compilados, e serão demonstrados em tabelas, quadros e percentuais, além de observar fraquezas metodológicas e pontos que necessitem de mais pesquisas e estudos futuros. Por fim, para proteger a validade da revisão integrativa, a pesquisadora elaborou suas conclusões e inferências, explicitando os vieses encontrados. 


\section{Resultados}

Na busca inicial foram encontrados 157 artigos somando-se todas as bases de dados. Após a leitura dos títulos dos artigos, notou-se que 10 deles se repetiram nas diferentes bases de pesquisa. Dos 147 artigos restantes, 133 foram excluídos após a leitura do título e do resumo, porque não se encaixavam nos critérios de inclusão apresentando objetivos diferentes do proposto pelo presente estudo. Foram pré-selecionados 14 artigos para avaliação do nível de evidência científica.

Quadro 1. Quantitativo de artigos encontrados nas diferentes bases de dados analisadas.

\begin{tabular}{|l|c|c|c|c|c|c|c|c|}
\hline & & PubMed & SciELO & $\begin{array}{c}\text { Portal } \\
\text { CAPES }\end{array}$ & Lilacs & MEDLINE & Cochrane & PEDro \\
\cline { 3 - 9 } $\begin{array}{c}\text { "Disfunçóes" } \\
\text { "Assoalho } \\
\text { pélvico", } \\
\text { "Radioterapia" } \\
\text { "Neoplasias do } \\
\text { colo do útero" }\end{array}$ & $\begin{array}{c}\text { Quantidade } \\
\text { encontrada }\end{array}$ & 34 & 25 & 78 & 3 & 14 & 3 & 0 \\
& $\begin{array}{c}\text { Descartados } \\
\text { Pré- }\end{array}$ & 31 & 20 & 76 & 2 & 12 & 2 & 0 \\
\hline $\begin{array}{c}\text { selecionados } \\
\text { Inclú́dos }\end{array}$ & 1 & 4 & 1 & 1 & 0 & 0 & 0 \\
\hline
\end{tabular}

Fonte: Autores.

Em um segundo momento os artigos pré-selecionados foram classificados de acordo com os níveis de hierarquia das evidências científicas, dos 14 artigos pré-selecionados foram incluídos na pesquisa 7 estudos, os outros 7 artigos foram excluídos, por se encaixarem nos níveis 5 e 6 de evidências científicas, correspondendo a dados provenientes de relatos de caso ou experiência e evidências baseadas nas opiniões de especialistas. Ao final da análise dos artigos, foi elaborado um fluxograma baseado no modelo PRISMA para todo o processo de seleção dos estudos (Fluxograma 1). 
Fluxograma 1. Fluxo de informações baseado no modelo PRISMA com o resultado da seleção dos artigos.

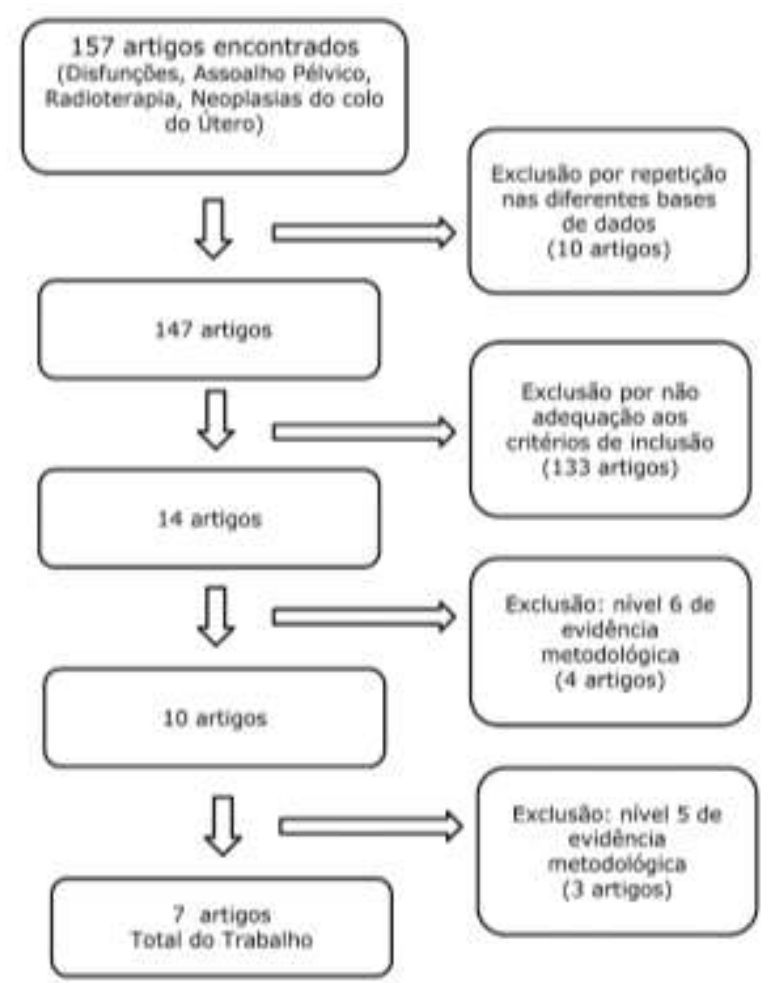

Fonte: Autores.

O Quadro 2 representa a condensação dos artigos selecionados durante a busca dos termos "Disfunções", "Assoalho pélvico", "Radioterapia" e "Neoplasias do colo do útero" e suas correlatas em inglês e espanhol, permanecendo sete estudos que se adequaram aos critérios da pesquisa. Em relação aos níveis de evidência, 1 artigo obteve dados através de estudo individual com delineamento experimental, 1 artigo com evidências provenientes de estudo quase-experimental e 5 artigos com evidências de estudos descritivos (não-experimentais) ou abordagem qualitativa. A idade das mulheres acometidas pelo câncer de colo de útero nos estudos incluídos variou de 26 a 92 anos. As disfunções pélvicas mais prevalentes, encontradas em três estudos foram a dispareunia e a incontinência urinária.

Quadro 2: Dados referentes aos artigos resultantes do cruzamento das palavras chaves "Disfunções", "Assoalho pélvico", "Radioterapia" e "Neoplasias do colo do útero".

\begin{tabular}{|c|c|c|c|c|c|c|}
\hline \multirow[t]{2}{*}{ Autor e ano } & \multirow[t]{2}{*}{ Objetivo } & \multirow{2}{*}{$\begin{array}{c}\text { Nível de } \\
\text { Evidência }\end{array}$} & \multicolumn{3}{|c|}{ Metodologia } & \multirow[t]{2}{*}{ Resultados } \\
\hline & & & Grupo & $\begin{array}{l}\text { Instrumento de } \\
\text { avaliação }\end{array}$ & $\begin{array}{l}\text { Avaliação ou } \\
\text { Intervenção }\end{array}$ & \\
\hline $\begin{array}{l}\text { Aguiar } \\
2015\end{array}$ & $\begin{array}{l}\text { Analisar } \\
\text { resultados os } \\
\text { terapêuticos em } \\
\text { pacientes tratados } \\
\text { com critérios } \\
\text { curativos por } \\
\text { meio } \\
\text { exclusivamente } \\
\text { de radioterapia } \\
\text { (RT) ou } \\
\text { radioquimio- } \\
\text { terapia (RQT). }\end{array}$ & Nível 2 & $\begin{array}{l}164 \\
\text { pacientes }\end{array}$ & $\begin{array}{lr}\text { Estadiamento de } \\
\text { acordo com a } \\
\text { FIGO (Federação } \\
\text { Internacional de } \\
\text { Ginecologia r e } \\
\text { Obstetrícia) } \\
\text { Imagens } \\
\text { radiológicas } \\
\text { provenientes de } \\
\text { tomografias } \\
\begin{array}{l}\text { computadoriza- } \\
\text { das. }\end{array}\end{array}$ & $\begin{array}{l}\begin{array}{l}\text { Radioterapia } \\
\text { combinada Externa } \\
\text { quimioterapia, com } \\
\text { dose }\end{array} \\
\text { variando de 46,8-50,4 Gy, } \\
\text { com reforço parametrial } \\
\text { de 14 Gy, todas as } \\
\text { pacientes foram irradiadas } \\
\text { com um acelerador linear } \\
\text { com cinco frações por } \\
\text { semana com dose diária de } \\
\text { 1,8 Gy e } 2 \text { Gy parametrial. } \\
\text { Concomitantemente a } \\
\text { quimioterapia foi feita }\end{array}$ & $\begin{array}{l}\text { De todas as } 164 \\
\text { pacientes, } 103 \\
\text { pacientes }(64 \%) \\
\text { também receberam } \\
\text { quimioterapia, à } \\
\text { base de cisplatina } \\
\text { semanal, com média } \\
\text { de } 4,7 \text { ciclos por } \\
\text { paciente. O tempo } \\
\text { médio total de } \\
\text { tratamento foi de } 58 \\
\text { dias. A sobrevida } \\
\text { global foi de } 67 \%\end{array}$ \\
\hline
\end{tabular}




\begin{tabular}{|c|c|c|c|c|c|}
\hline & & & $\begin{array}{l}\text { Taxa de controle } \\
\text { loco-regional, } \\
\text { sobrevida a cinco } \\
\text { anos } \\
\text { complicações } \\
\text { crônicas através } \\
\text { do RTOG e } \\
\text { EORTC. }\end{array}$ & $\begin{array}{l}\text { com base na cisplatina em } \\
\text { doses de } 40 \mathrm{mg} / \mathrm{m}^{2} \text {. } \\
\text { Braquiterapia (BT) } \\
\text { Realizada com } \\
\text { equipamento de média } \\
\text { dose (Curietron) de uso } \\
\text { ginecológico exclusivo. O } \\
\text { equipamento possui cinco } \\
\text { fontes de Césio-137, três } \\
\text { para colocações } \\
\text { intrauterinas e } 2 \text { fontes } \\
\text { vaginais. Foram aplicadas } \\
\text { duas doses de BT no } \\
\text { mesmo dia, com intervalo } \\
\text { de pelo menos } 6 \text { horas } \\
\text { entre elas, a segunda } \\
\text { intervenção foi realizada } \\
\text { com no mínimo } 7 \text { dias de } \\
\text { intervalo. A dose } \\
\text { fracionada prescrita no } \\
\text { ponto A foi de } 610 \text { Gy } \\
\text { para tumores menores que } \\
5 \text { cm e } 660 \text { Gy para } \\
\text { tumores iguais ou maiores } \\
\text { que } 5 \text { cm, com limitações } \\
\text { de dose no reto e bexiga. }\end{array}$ & $\begin{array}{l}\text { em cinco anos, } \\
\text { mostrando } \\
\text { diferenças } \\
\text { significativas entre o } \\
\text { estágio II (78\%) e o } \\
\text { estágio III b (49\%) } \\
\text { ao longo de todo o } \\
\text { tempo analisado (p = } \\
0,0002) \text {. A taxa de } \\
\text { complicaçôes nos } \\
\text { graus 3-4 RTOG foi: } \\
\text { urinária 1,8\% (3 } \\
\text { casos) e digestiva } \\
3,7 \% \text { (6 casos). O } \\
\text { controle local inicial } \\
\text { foi de 89\% e a } \\
\text { persistência da lesão } \\
\text { de } 10,3 \% \text { recidiva } \\
\text { loco-regional (RL) } \\
\text { em todo o período: } \\
19,5 \% \text { metástases } \\
\text { com ou sem RL: } \\
10,3 \%\end{array}$ \\
\hline $\begin{array}{l}\text { Castaneda \&, } \\
2019\end{array}$ & $\begin{array}{lrl}\text { Verificar } & \text { a } & \text { Nível } 4 \\
\text { prevalência } & \text { de } & \\
\text { incapacidade } \mathrm{em} & \\
\text { mulheres com } & \\
\text { câncer do colo do } & \\
\text { útero } \quad(\mathrm{CCU}) & \\
\text { utilizando } & \mathrm{a} \\
\text { Classificação } & \\
\text { Internacional de } \\
\text { Funcionalidade, } \\
\text { Incapacidade e } \\
\text { Saúde (CIF). }\end{array}$ & $\begin{array}{l}116 \\
\text { pacientes }\end{array}$ & $\begin{array}{l}\text { O instrumento de } \\
\text { coleta de dados foi } \\
\text { composto por } \\
\text { questões } \\
\text { sociodemográ- } \\
\text { ficas, informações } \\
\text { clínicas, checklist } \\
\text { da CIF e o } \\
\text { questionário de } \\
\text { qualidade de vida } \\
\text { Functional } \\
\text { Assessment of } \\
\text { Cancer Therapy } \\
\text { (FACT-Cx). }\end{array}$ & $\begin{array}{l}\text { Essas mulheres foram } \\
\text { avaliadas através do } \\
\text { questionário da CIF, esse } \\
\text { instrumento apresenta } 29 \\
\text { categorias de funções do } \\
\text { corpo, } 16 \text { de estruturas do } \\
\text { corpo, } 48 \text { de atividade e } \\
\text { participação, e por fim } 31 \\
\text { de fatores ambientais. } \\
\text { Outro fator analisado foi a } \\
\text { qualidade de vida dessas } \\
\text { mulheres através do } \\
\text { FACT-Cx que determina a } \\
\text { funcionalidade e a } \\
\text { condição da mulher com } \\
\text { câncer de colo de útero, } \\
\text { apresentando como } \\
\text { referência temporal os } \\
\text { últimos sete dias. O } \\
\text { FACT-Cx consta de } 42 \\
\text { itens distribuídos da } \\
\text { seguinte maneira: } 27 \text { itens } \\
\text { relacionados ao módulo } \\
\text { geral divididos nos } \\
\text { domínios de bem-estar } \\
\text { físico, social/familiar, } \\
\text { funcional e bem-estar } \\
\text { emocional; os demais } 15 \\
\text { itens se referem aos } \\
\text { domínios relacionados aos } \\
\text { sintomas específicos da } \\
\text { área ginecológica, } \\
\text { intestinal, urinária, } \\
\text { alterações vaginais, } \\
\text { preocupação com o } \\
\text { tratamento, alimentação, } \\
\text { sexualidade escore } \\
\text { autoimagem. O es } \\
\text { varia de } 0 \text { a } 168, \text { e quanto } \\
\text { mais elevada a pontuação, }\end{array}$ & $\begin{array}{l}\text { A maior parte foi } \\
\text { diagnosticada no } \\
\text { estádio IB, e mais da } \\
\text { metade recebeu } \\
\text { como tratamento a } \\
\text { quimioterapia } \\
\text { conjugada com } \\
\text { radioterapiar A } \\
\text { maior prevalência } \\
\text { de incapacidade foi } \\
\text { encontrada para a } \\
\text { deficiência das } \\
\text { estruturas do } \\
\text { sistema reprodutivo } \\
\text { (96,6\%), seguido de } \\
\text { problemas nas } \\
\text { funções da energia e } \\
\text { do impulso (70,3\%), } \\
\text { funções emocionais } \\
\text { (70,7\%) e limitação } \\
\text { para carregar e } \\
\text { transportar } \\
\text { objetos (67,2\%). O } \\
\text { domínio do bem- } \\
\text { estar emocional foi o } \\
\text { mais comprometido } \\
\text { de acordo com a } \\
\text { análise realizada } \\
\text { pelo FACT-Cx. A } \\
\text { prevalência de } \\
\text { incapacidade em } \\
\text { mulheres com CCU } \\
\text { foi elevada para } \\
\text { todos or os } \\
\text { componentes da } \\
\text { CIF, refletindo o } \\
\text { impacto tanto da } \\
\text { doença quanto do } \\
\text { seu tratamento na } \\
\text { funcionalidade. }\end{array}$ \\
\hline
\end{tabular}




\begin{tabular}{|c|c|c|c|c|c|}
\hline & & & & & $\begin{array}{l}\text { melhor a qualidade de vida } \\
\text { relacionada à saúde. }\end{array}$ \\
\hline $\begin{array}{l}\text { Correia \&, } \\
2020 .\end{array}$ & $\begin{array}{l}\text { Descrever as } \\
\text { características } \\
\text { sociodemográfi- } \\
\text { cas, clínicas e } \\
\text { relacionadas à vida } \\
\text { sexual e identificar } \\
\text { a disfunção sexual } \\
\text { em mulheres após } \\
\text { o tratamento do } \\
\text { câncer do colo do } \\
\text { útero. }\end{array}$ & Nível 4 & 46 pacientes & $\begin{array}{l}\text { Questionário } \\
\text { sociodemográ- } \\
\text { fico e clínico } \\
\text { construído pelas } \\
\text { próprias autoras. } \\
\text { Îndice de Função } \\
\text { Sexual (FSFI). }\end{array}$ & 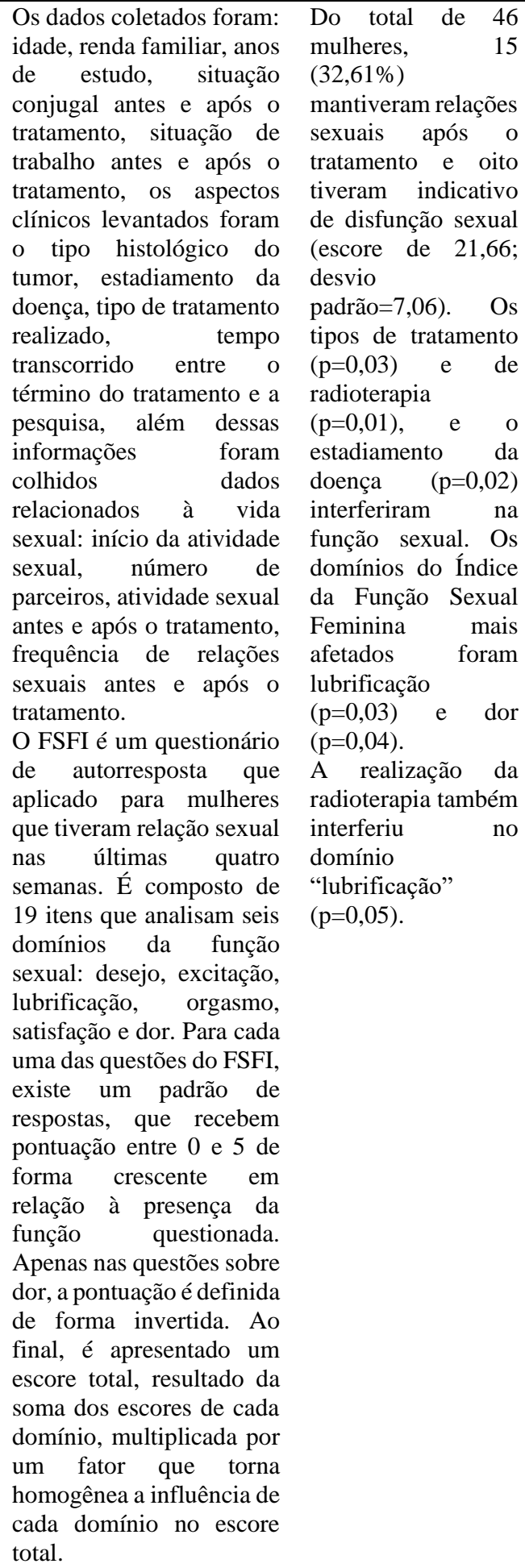 \\
\hline $\begin{array}{l}\text { Hazewinkel } \\
\&, 2012\end{array}$ & $\begin{array}{l}\text { Identificar } \\
\text { associações entre } \\
\text { variáveis } \\
\text { demográficas, } \\
\text { relacionadas com a } \\
\text { doença e variáveis } \\
\text { psicológicas e } \\
\text { sofrimento grave } \\
\text { de sintomas do } \\
\text { assoalho pélvico }\end{array}$ & ível 4 & $\begin{array}{l}282 \\
\text { pacientes }\end{array}$ & $\begin{array}{l}\text { Inventário de } \\
\text { angústia } \\
\text { urogenital } \\
\text { validado para o } \\
\text { idioma holandês } \\
\text { (UDI) e o } \\
\text { inventário de } \\
\text { sofrimento } \\
\text { defecatório (DDI), } \\
\text { além disso foram }\end{array}$ & $\begin{array}{lrrlr}\text { A população } & \text { do } & \text { A menor pontuação } \\
\text { estudo em } 3 \text { grupos: } & \text { mediana para imagem } \\
\text { pacientes tratados } & \text { corporal, indicando pouca } \\
\text { com } & (1) & \text { perturbação da imagem } \\
\text { histerectomia } & & \text { corporal, foi relatada pelo } \\
\text { radical (RH) } & \text { e } & \text { grupo PRT. Sofrimento } \\
\text { dissecção } & \text { do } & \text { severo de incontinência } \\
\text { linfonodo pélvico } & \text { urinária e micção } \\
(\text { LND) (HR + LND), } & \text { obstrutiva foi relatado por } \\
(2) \quad \text { cirurgia } & \text { e } & \text { 24\% a 36\% dos pacientes }\end{array}$ \\
\hline
\end{tabular}




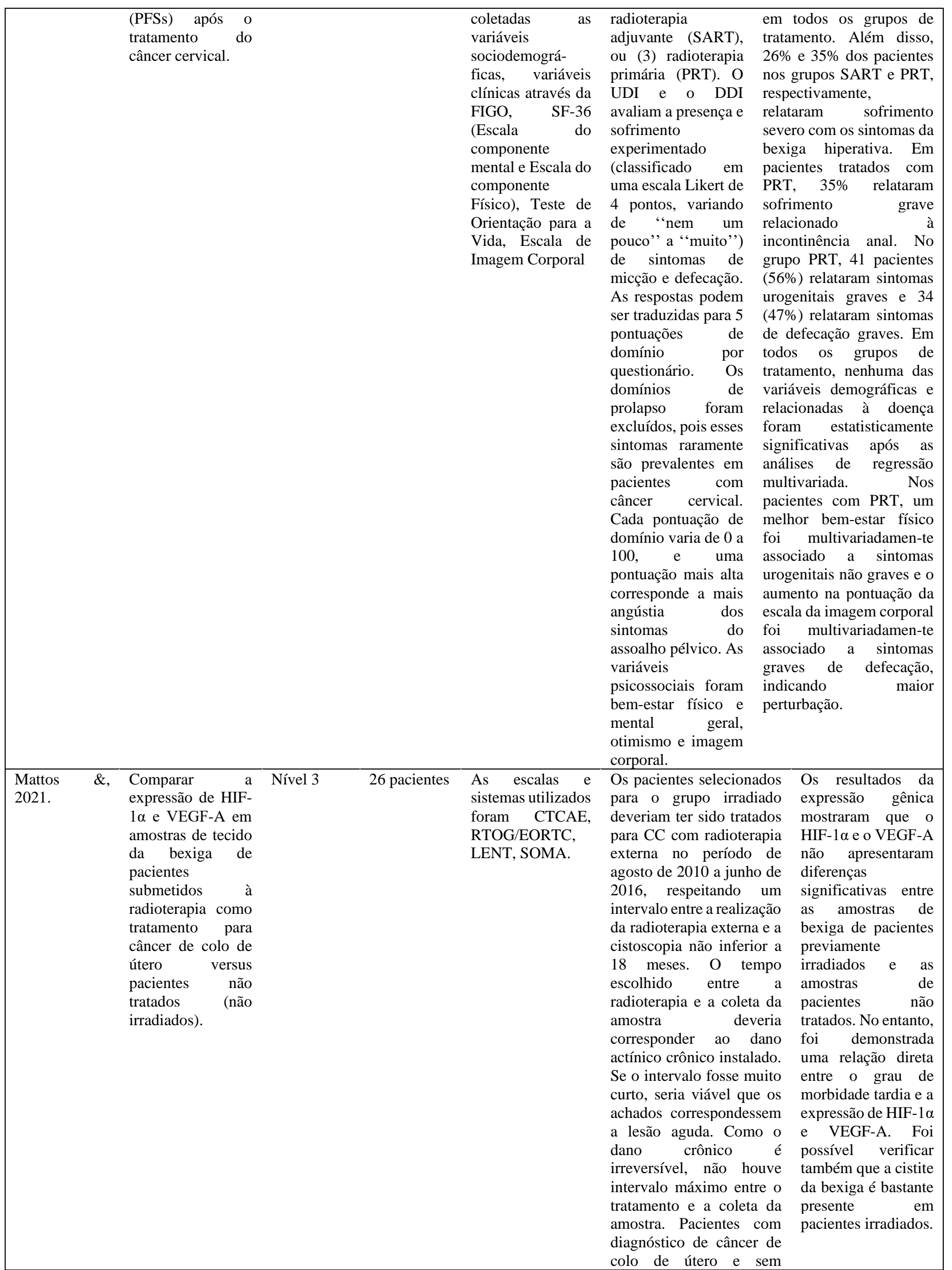




\begin{tabular}{|c|c|c|c|c|}
\hline & & & & $\begin{array}{l}\text { tratamento, cujo } \\
\text { estadiamento clínico não } \\
\text { era mais avançado do que } \\
\text { IIIA foram incluídos no } \\
\text { grupo controle. Todos os } \\
\text { dados demográficos, } \\
\text { clínicos e laboratoriais } \\
\text { relevantes, tipo } \\
\text { histológico, estadiamento } \\
\text { e doses de radiação foram } \\
\text { coletados dos prontuários } \\
\text { médicos e um exame } \\
\text { endoscópico da bexiga foi } \\
\text { realizado. }\end{array}$ \\
\hline $\begin{array}{l}\text { Menezes } \\
2017 .\end{array}$ & $\begin{array}{llr}\text { \&, } & \text { Avaliar as } & \text { disfu } \\
\text { nções do assoal } \\
\text { ho pélvico cons } \\
\text { equente } & \text { ao } \\
\text { tratamento } & \\
\text { de câncer do col } \\
\text { o do útero. }\end{array}$ & Nível $4 \quad 12$ pacientes & $\begin{array}{l}\text { Ficha de avalia } \\
\text { ção com dados } \\
\text { sociodemográfico } \\
\text { s e clínicos, } \\
\text { questionário } \\
\text { contendo } \\
\text { perguntas so- } \\
\text { bre a intensidade } \\
\text { de dor durante } \\
\text { a relação sexual } \\
\text { que foram respo } \\
\text { ndidos de forma } \\
\text { objetiva, além da } \\
\text { Escala Visual } \\
\text { Analógica, } \\
\text { avaliação } \\
\text { disfunção de } \\
\text { miccional, além } \\
\text { do Pad Test, caso } \\
\text { necessário } \\
\text { avaliação } \\
\text { funcional } \\
\text { assoalho pélvico } \\
\text { (AFA) foi medida } \\
\text { pela palpação bi- } \\
\text { digital. }\end{array}$ & 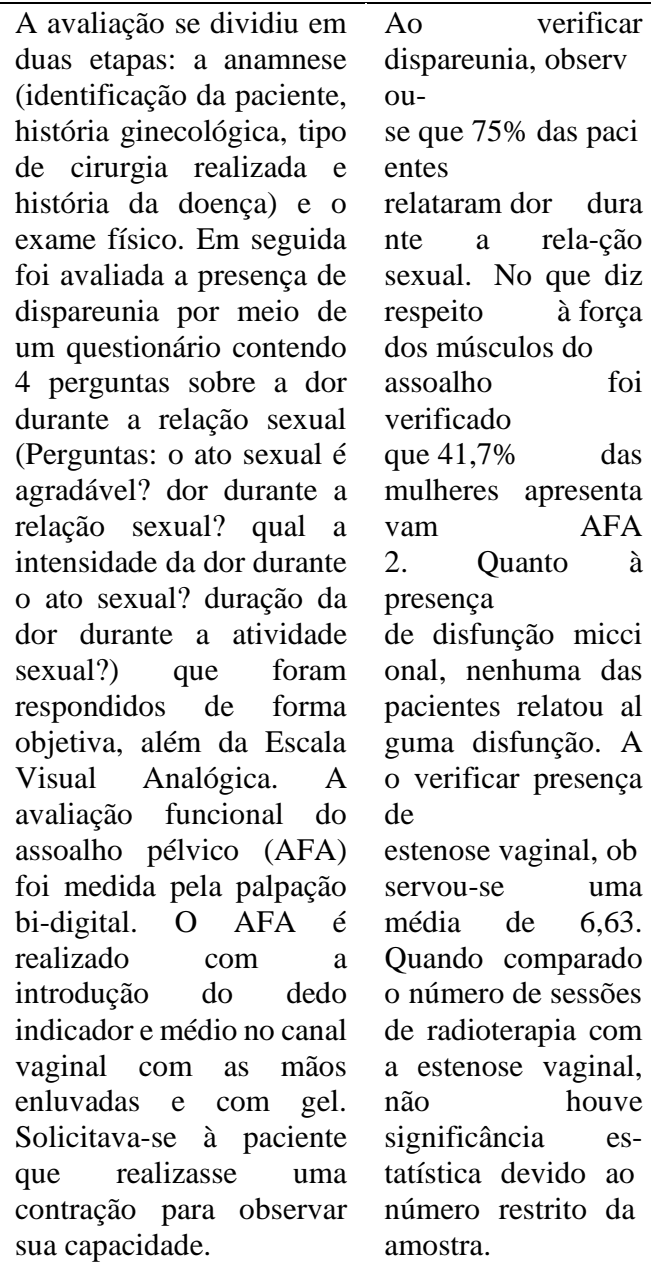 \\
\hline $\begin{array}{l}\text { Miguel } \\
2020 .\end{array}$ & $\begin{array}{ll}\text { \&, } & \text { Avaliar o impacto } \\
\text { da } & \\
\text { quimiorradiação } \\
\text { na r função } \\
\text { muscular } & \text { do } \\
\text { assoalho pélvico } \\
\text { (AP) após o } \\
\text { tratamento do do } \\
\text { câncer cervical } \\
\text { (CC). }\end{array}$ & Nível 4 & $\begin{array}{l}\text { Avaliação da } \\
\text { função muscular } \\
\text { através } \\
\text { perineometria da } \\
\text { (PNM) e } \\
\text { eletromiogra-fia } \\
\text { de superfície } \\
(\mathrm{EMG}) .\end{array}$ & 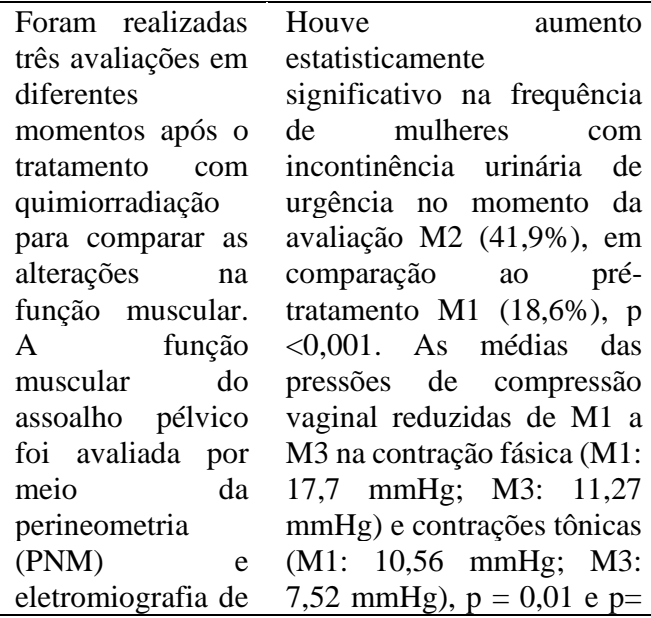 \\
\hline
\end{tabular}




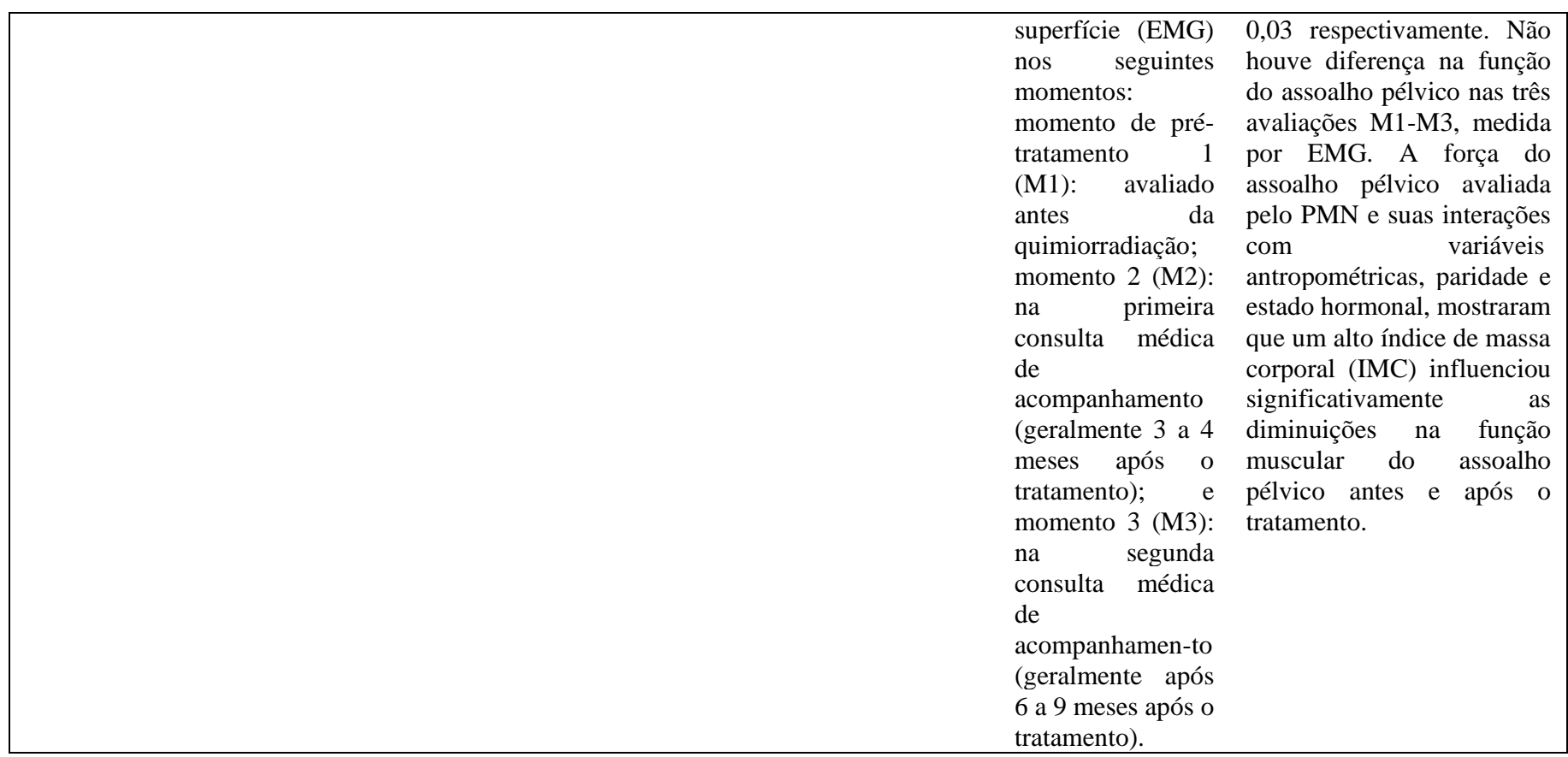

Fonte: Autores.

\section{Discussão}

O presente estudo verificou que entre as disfunções do assoalho pélvico promovidas pela radioterapia para o tratamento de carcinoma de colo uterino destacam-se a dispareunia e a incontinência urinária. A redução da mortalidade pelas neoplasias de colo uterino traz uma série de novos desafios para os profissionais de saúde, relacionados principalmente pelas sequelas dos tratamentos adotados. Os diferentes recursos terapêuticos utilizados repercutem com diversos impactos no assoalho pélvico. Em relação a radioterapia é possível verificar diversos prejuízos na funcionalidade dessas mulheres e distintos impactos na sua qualidade de vida. (Bae \&, 2016).

A musculatura do assoalho pélvico desempenha um importante papel na manutenção das funções dos órgãos localizados nessa região, além de servir de suporte para essas estruturas, a contração desse grupamento muscular atua diretamente em atividades como a continência urinária e fecal. A radioterapia pode trazer diversos transtornos para esses músculos. Os estudos de Menezes e colaboradores (2017) e Miguel e seus contribuintes (2020) descrevem a fraqueza muscular após a conclusão do tratamento. O primeiro utilizou a Avaliação Funcional do Assoalho Pélvico (AFA), que se caracteriza pela palpação bidigital para avaliar a força de contração desses músculos, já no estudo de coorte prospectiva de Miguel et al., essa avaliação foi feita antes, durante e após a conclusão do tratamento através da perioneometria.

Outras diversas alterações do assoalho pélvico foram elucidadas como sequelas após a radioterapia, a dispareunia é um tipo de disfunção sexual e foi relatada nos estudos descritivos de Castaneda e colaboradores (2019); Menezes, et al. (2017) e Correia e colaboradores (2020). Essa disfunção se caracteriza por dor durante a relação sexual, nesses trabalhos a alteração foi mensurada através de distintos recursos avaliativos, no primeiro foi utilizada a Classificação Internacional de Incapacidade, Funcionalidade e Saúde (CIF) da Organização Mundial de Saúde (OMS) e o questionário de qualidade de vida Functional Assessment of Cancer Therapy (FACT-Cx). No estudo de Menezes e colaboradores (2017) foi utilizado um questionário específico para dispareunia que contava com 4 perguntas acerca do ato sexual, com enfoque na dor, sua duração e intensidade, em contrapartida o estudo de Correia utilizou o Índice de Função Sexual (FSFI). Nos estudos de Castaneda, et al. e Menezes, et al. também é evidenciada a estenose vaginal, após a radioterapia, que corresponde ao encurtamento do canal vaginal em decorrência da radiação. 
As alterações na função urinária e digestiva também se mostraram frequentes após o tratamento com radioterapia para neoplasia do colo de útero, sendo relatadas nos estudos de Aguiar et al. (2015), Castaneda, et al. (2019), Mattos, et al. (2021), Hazewinkel, et al. (2012) e Miguel, et al. (2020), há um destaque para a presença de constipação e incontinência urinária nestas pacientes. Outras alterações encontradas também foram a cistite induzida por radiação, micção obstrutiva e a incontinência fecal. Entretanto, apenas 2 desses estudos considerou o impacto dessas alterações no bem-estar físico e emocional dessas mulheres e suas consequentes perturbações na qualidade de vida. Dentro do estudo de Hazewinkel e colaboradores foi possível inferir que uma pior qualidade de vida estava atrelada a participantes com sintomas urogenitais graves.

As mulheres submetidas a esse tratamento devem ser vistas como seres biopsicossociais, pensar sobre a funcionalidade durante e após a conclusão do tratamento é de extrema importância, nesse sentido a fisioterapia pode atuar tanto de forma preventiva para a não instalação de algumas dessas incapacidades, como buscar a promoção de saúde e a reabilitação de disfunções já instaladas. Castaneda e colaboradores (2015) propõem a inclusão de programas de intervenção variados visando a individualidade de cada paciente.

Na presente revisão integrativa, observou-se que o único estudo com evidências experimentais não cumpriu critérios de alocação aleatória e secreta, administração da terapia e mensuração dos resultados de forma cega. Não foi encontrado nenhum artigo com nível 1 de evidência, que se caracteriza por evidências de alta qualidade metodológica com dados provenientes da meta-análise de múltiplos estudos clínicos controlados e randomizados. Algumas limitações dos estudos incluídos na pesquisa são um grupo restrito de participantes, na maior parte dos casos as mulheres eram atendidas nos serviços onde as pesquisas foram desenvolvidas, a ausência de cálculo amostral e a não uniformização dos padrões avaliativos. A escassez de dados provenientes de evidências nível 1 e 2 também revela a necessidade de mais pesquisas nessa área que considerem todos os critérios metodológicos para garantir confiabilidade à pesquisa e aos dados encontrados.

\section{Considerações Finais}

A presente revisão integrativa teve a finalidade de examinar as disfunções pélvicas que surgem após o tratamento através da radioterapia para o câncer de colo do útero. Foi possível inferir que esse tipo de intervenção traz inúmeros impactos para o assoalho pélvico, com destaque para a incontinência urinária e a dispareunia. Contudo, mostra-se que são necessários mais estudos de alta qualidade metodológica que abordem esse tema, apontando outras alterações pélvicas que também afetem a saúde das pessoas acometidas por essa patologia. Estudos que avaliem o impacto das disfunções no assoalho pélvico são imprescindíveis, pois essas sequelas afetam as atividades de vida diária dessa população, além de impactar nas suas relações interpessoais e no bem-estar mental e físico.

Sugere-se a partir dos resultados, trabalho que abordem a inserção de forma mais ampla de atividades de recuperação através da fisioterapia para as alterações decorrentes desse tipo de tratamento, visando a melhora da funcionalidade dos músculos do assoalho pélvico e redução das alterações pélvicas, possibilitando assim uma melhora da qualidade de vida dessa população. Para maior confiabilidade dos resultados e redução dos vieses recomenda-se a utilização de cálculo amostral, utilização de grupo controle e intervenção, de forma cega tanto para os participantes da pesquisa, quanto para os pesquisadores.

\section{Referências}

Aguiar, D. S., Rosa, M. D., Rosa, A., Lilio, A. C. O., Ronco, A., Mara, C., Paolini, G., Guerrero, L., Ferreira, V., Silvera, J., Terzieff, V., Alonso, I., Blanco, A. \& Quarneti, A. (2015). Tratamiento con radioquimioterapia del cáncer de cuello uterino: resultados a largo plazo. Revista Médica del Uruguay. 31(4), 2418. http://www.scielo.edu.uy/scielo.php?script=sci_arttext\&pid=S1688-03902015000400003\&lng=es\&tlng=es.

Bae, H. \& Park, H. (2016). Sexual function, depression, and quality of life in patients with cervical cancer. Support Care Cancer. 24,(24), 1277-83. https://doi.org/10.1007/s00520-015-2918-z. 
Bernado, B. C., Lorenzato, F. R. B., Figueiroa, J. N. \& Kitoko, P. M. (2007). Disfunção sexual em pacientes com câncer do colo uterino avançado submetidas

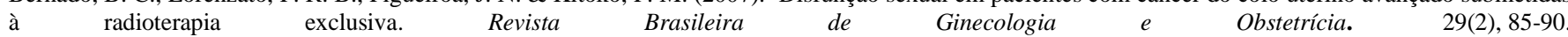
https://www.scielo.br/j/rbgo/a/JvcYhBNFGr8mQzV7LqqGk9z/?format=pdf.

Castaneda, L., Bergmann, A., Castro, S. \& Koifman, R. (2018). Functioning in women with cervical cancer in Brazil: the perspective of experts. Revista Brasileira de Ginecologia e Obstetrícia. 40(5), p. 260-265. https://doi.org/10.1055/s-0038-1646921.

Castaneda, L., Bergmann, A., Castro, S. \& Koifman, R. (2019). Prevalência de incapacidades e aspectos associados em mulheres com câncer de colo do útero, Rio de Janeiro, Brasil. Cadernos Saúde Coletiva. 27(3), 307-15. https://doi.org/10.1590/1414-462X201900030440.

Corrêa, C. S. L., Leite, I. C. G., Andrade, A. P. S., Ferreira, A. S. S., Carvalho, S. M. \& Guerra, M. R. (2016). Sexual function of women surviving cervical cancer. Archives of Gynecology and Obstetrics. 293(3), 1053-63. https://doi.org/10.1007/s00404-015-3857-0.

Correia, R. A., Bonfim, C. V., Ferreira, D. K. S., Furtado, B. M. A. S. M., Costa, H. V. V., Feitosa, K. M. A., \& Santos, S. L. (2020). Quality of life after treatment for cervical cancer. Escola de Enfermagem Anna Nery - EEAN. 22(4):e20180130. https://doi.org/10.1590/S1980-220X2019029903636.

Einstein, M. H., Rash, J., Chappel, R., Swietlik, J., Hollenberg, J. \& Connor, J. P. (2012). Quality of life in cervical cancer survivors: Patient and provider perspectives on common complications of cervical cancer and treatment. Gynecologic Oncology. 125(1), 163-7. https://doi.org/10.1016/j.ygyno.2011.10.033.

Ferla, L., Darski, C, Paiva, L. L., Sbruzzi, G. \& Vieira, A. (2016). Synergism between abdominal and pelvic floor muscles in healthy women: a systematic review of observational studies. Fisioterapia em Movimento. 29(2), 399-410. • https://doi.org/10.1590/0103-5150.029.002.AO19.

Freire, G. M., Dias, R. S., Giordani, A. J., Ribalta, J. C. L., Segreto, H. R. C. \& Segreto, R. A. (2010). Ressonância magnética para avaliação dos limites dos campos clássicos de radioterapia em pacientes portadoras de neoplasia maligna de colo uterino. Radiologia Brasileira. 43(3), 175-8. https://www.scielo.br/j/rb/a/CRwBjmYwqLPwXJywqKX659k/?lang=pt\&format=pdf.

Fonseca, A. J. da, Ferreira, L. P., Dalla-benetta, A. C., Roldan, C. N. \& Ferreira, M. L. S. (2010). Epidemiologia e impacto econômico do câncer de colo de útero no Estado de Roraima: a perspectiva do SUS. Revista Brasileira de Ginecologia e Obstetrícia. 32(8), 386-92. https://doi.org/10.1590/S010072032010000800005 .

Hazewinkel, M. H., Sprangers, M. A. G., Velden, J. V.D., Burger, M. P. M. \& Roovers, J. P. W. R. (2012). Severe Pelvic Floor Symptoms After Cervical Cancer Treatment Are Predominantly Associated With Mental and Physical Well-Being and Body Image: A Cross-Sectional Study. International Journal of Gynecological Cancer. 22(1), 154-60 . https://doi.org/10.1097/IGC.0b013e3182332df8.

Instituto Nacional do Câncer José Alencar Gomes da Silva (INCA). (2021). Câncer de colo de útero. https://www.inca.gov.br/tipos-de-cancer/cancer-do-colodo-utero.

Instituto Nacional de Câncer José Alencar Gomes da Silva (INCA). (2019). Estimativa 2020: incidência de câncer no Brasil. https://www.inca.gov.br/sites/ufu.sti.inca.local/files/media/document/estimativa-2020-incidencia-de-cancer-no-brasil.pdf.

Mattos, R. C., Guimarães, I. S., Souza, L. T. \& Melo, A. C. (2021). Evaluation of HIF-1 $\alpha$ and VEGF-A expression in radiation induced cystitis: A case-control study. International Brazilian Journal of Urology. 47(2) 295-305. https://doi.org/10.1590/S1677-5538.IBJU.2020.0054.

Menezes, E. T. T., Rodrigues, R. D. S., Pontes, L. S., Dias, G. A. S., Latorre, G. F. S. \& Nunes, E. F. C. (2017). Avaliação fisioterapêutica nas disfunções do assoalho pélvico consequente ao tratamento de câncer do colo do útero. Fisioterapia Brasil. 18(2), 189-196. https://pesquisa.bvsalud.org/portal/resource/pt/biblio-884406.

Miguel, T. P., Laurienzo, C. E., Faria, E. F., Sarri, A. J., Castro, I. Q., Affonso-Júnior, R. J., Andrade, C. E. M. C., Vieira, M. A. \& Reis, R. (2020). Chemoradiation for cervical cancer treatment portends high risk of pelvic floor dysfunction. Plos One. 15(6), 1-12. https://doi.org/10.1371/journal.pone.0234389.

Oliveira, A. C. Z., Esteves, S. C. B., Feijó, L. F. A., Tagawa, E. K. \& Cunha, M. O. (2005). Interstitial high-dose rate brachytherapy for recurrent cervical cancer after radiation therapy. Radiologia Brasileira. 38(2), 117-120. https://www.researchgate.net/publication/262652359_Interstitial_highdose_rate_brachytherapy_for_recurrent_cervical_cancer_after_radiation_therapy.

Osann, K., Hsieh, S.; Nelson E. L., Monk, B. J., Chase, D., Cella, D. \& Wenzel, L. (2014). Factors associated with poor quality of life among cervical cancer survivors: implications for clinical care and clinical trials. Gynecologic Oncology, 135(2):266-72. https://doi.org/10.1016/j.ygyno.2014.08.036.

Pfaendler, K. S., Wenzel, L., Mechanic, M. B. \& Penner, K. R.(2015). Cervical cancer survivorship: long-term quality of life and social support. Clinical Therapeutics. 37(1), p.39-48. http://dx.doi.org/10.1016/j.clinthera.2014.11.013.

Ros, C. \& Espuna, M. (2013). Impacto del tratamiento del cáncer de cérvix sobre la función miccional y sexual. Actas Urológicas Espanholas. 37(1) 40-6. https://doi.org/10.1016/j.acuro.2012.03.008.

Sacomori, C., Virtuoso, J. F., Kruger, A. P. \& Cardoso, F. L. (2015). Pelvic floor muscle strength and sexual function in women. Fisioterapia em Movimento. 28(4), 657-65. https://doi.org/10.1590/0103-5150.028.004.AO02. 
Research, Society and Development, v. 10, n. 14, e356101422036, 2021

(CC BY 4.0) | ISSN 2525-3409 | DOI: http://dx.doi.org/10.33448/rsd-v10i14.22036

Santos, L. N., Castaneda, L., Aguiar, S. S., Thuler, L. C. S., Koifman, R. J. \& Bergmann, A. (2019). Health-related Quality of Life in Women with Cervical Cancer. Revista Brasileira de Ginecologia e Obstetrícia. 41 (4), 242-8. https://doi.org/10.1055/s-0039-1683355.

Souza, L. M., Pegorare, A. B. G., Christofoletti, G. \& Barbosa, S. R. M. (2017). Influence of a protocol of Pilates exercises on the contractility of the pelvic floor muscles of non-institutionalized elderly persons. Revista Brasileira de Geriatria e Gerontologia. 20(4), 485-93. https://doi.org/10.1590/198122562017020.160191

Souza, M. T., Silva M. D. \& Carvalho, R. (2010). Revisão integrativa: o que é e como fazer. Einstein. 8(1), 102-6. https://journal.einstein.br/wpcontent/uploads/articles_xml/1679-4508-eins-S1679-45082010000100102/1679-4508-eins-S1679-45082010000100102-pt.pdf?x56956. 